\title{
Full spectrum optical constant interface to the Materials Project
}

\author{
J. J. Kas ${ }^{\mathrm{a}, \mathrm{b}}$, F. D. Vila ${ }^{\mathrm{a}, \mathrm{b}}$, C. D. Pemmaraju ${ }^{\mathrm{b}}$, M. P. Prange ${ }^{\mathrm{c}}$, K. A. Persson ${ }^{\mathrm{d}}$, R. X. Yang ${ }^{\mathrm{d}}$, J. J. Rehra,b,* \\ ${ }^{a}$ Department of Physics, University of Washington, Seattle, WA 98195, USA \\ ${ }^{b}$ Stanford Institute for Materials and Energies Sciences, SLAC National Accelerator Laboratory, Menlo Park, CA 94025, USA \\ ${ }^{c}$ Physical and Computational Sciences Directorate, Pacific Northwest National Laboratory: Richland, WA 99352 \\ ${ }^{d}$ Materials Science Division, Lawrence Berkeley National Laboratory, Berkeley, CA 94720, USA
}

\section{Abstract}

Optical constants characterize the interaction of materials with light and are important properties in material design. Here we present a Python-based Corvus workflow for simulations of full spectrum optical constants from the UVD VIS to hard x-ray wavelengths based on the real-space Green's function code FEFF10 and structural data from the Materials Project (MP). The Corvus workflow manager and its associated tools provide an interface to FEFF10 and the MP database. The workflow parallelizes the FEFF computations of optical constants over all absorption edges for each material in the MP database specified by a unique MP-ID. The workflow tools determine the distribution of computational resources needed for that case. Similarly, the optical constants for selected sets of materials can be computed in a single-shot. To illustrate the approach, we present results for nearly all elemental solids in the periodic table, as well as a sample compound, and compared with experimental results. As in x-ray absorption spectra, these results are interpreted in terms of an atomic-like background and fine-structure contributions.

Keywords: Optical constants, Materials Project, FEFF, Corvus

\section{Introduction}

Optical constants characterize the frequency dependent interaction between light and matter in the longwavelength limit. Thus they are often important characteristics in materials design. These properties include the complex dielectric constant and index of refraction, as well as the energy-loss function, photoabsorption coefficient, and optical reflectivity. 11 4] Many other physical properties can be derived from the optical constants, including electron energy loss spectra (EELS), inelastic mean-free paths, the atomic scattering amplitudes, and Hamaker constants for the van der Waals interaction. For these purposes, tabulations of experimentally determined optical constants are widely used. 1-4 For practical reasons, however, such tabulations are limited to a relatively small number of well characterized materials over limited spectral ranges and limited environmental conditions. Thus here we focus on broad spectrum theoretical treatments for the systems defined in the Materials Project(MP) database.

Significant progress has been made in the fundamental theory of optical properties since the pioneering works of Nozieres and Pines, [5] Adler, [6] and Wiser. [7] In particular modern first-principles calculations based on time-dependent density functional theory (TDDFT) and the Bethe-SalpeterEquation (BSE) are now highly

\footnotetext{
${ }^{*}$ Corresponding author

Email address: jjr@uw.edu (J. J. Rehr)
}

quantitative. 86 17] Also limited tables of theoretical optical constants of materials have been compiled over the optical range. [18, 19] However, such calculations involve many-body calculations of optical response, and become computationally intractable for many materials over broad spectral ranges. In contrast, the real-space Green's function (RSGF) approach in FEFF10 includes the key manybody effects, is highly automated and has proved to be quite accurate for general materials in the x-ray regime. Moreover the code can be semi-quantitative in the UV-VIS to soft-x-ray regime. 20] Thus the code provides an efficient platform for calculations of dielectric properties over a broad spectral range. Consequently, theoretical calculations based on FEFF10 provide an attractive alternative to available theoretical and experimental tabulations of optical constants for many purposes. Nevertheless, selected experimental measurements are important to validate the theory.

Recently K- and L-shell XAS calculations based on the FEFF code have been added for a very large number of materials in the Materials Project (MP) database. 21, 22] These data have been exploited, e.g., in machine-learning models for the interpretation of XAS data. 21, 23, Our aim here is to complement these properties with a more complete set of optical constants for all edges from the UV-VIS to hard-x-ray energies. Consequently, the results presented here provide significant extension, both in the variety of optical-constant spectra, spectral range, and material properties. Our approach is based on the the devel- 
opment of the Corvus [opcons] workflow, where Corvus is the workflow engine, 24 and opcons is the target property. In particular, the approach imports the structural and property data from the MP, and then parallelizes and automates the calculations permitting high-throughput calculations of optical constants for materials throughout the MP database. Our procedure for theoretical calculations of optical constants over all edges essentially follows that described in detail by Prange et al.,20, but has been updated for the FEFF10 code. In addition, refinements for including vibrational disorder via the correlated Debye model using MP data have been added and the calculations for the UV-VIS range have been simplified. To illustrate the approach calculations have been carried out for nearly all elemental solids throughout the periodic table and explicit examples are given for $\mathrm{Cu}, \mathrm{Ag}$, and $\mathrm{Au}$, together with comparisons to experimental data. In addition we show results for a sample compound $\mathrm{Al}_{2} \mathrm{O}_{3}$ to validate our approximation in the UV-VIS range. The Corvus [opcons] workflow described here is applicable to any material available in the MP database as defined by a given MP identificatoin label (MP-ID), thus making possible routine calculations of optical constants for over $10^{5}$ structures. By default, the calculations are carried out with lattice vibration effects included at room temperature, while electronic temperature and thermal expansion effects are neglected. However, corrections for thermal expansion, finite temperature, and pressures other than 1 atm can be added using subsequent calculations that reuse some of the previously computed data. The workflow is naturally parallelized and, for typical settings, requires as little as minutes of wall-clock time per material on massively parallel systems.

\section{Optical constants with Corvus and FEFF10}

In general, the optical constants are related to the complex dielectric constant $\epsilon(\omega)=\epsilon_{1}(\omega)+i \epsilon_{2}(\omega)$ in the long wave-length limit, where $\omega$ is the frequency of the electromagnetic field. Since their real and imaginary parts are related by Kramers-Kronig (KK) transformations, they can all be calculated from the imaginary part $\epsilon_{2}(\omega)$ (e.g. Fig. 1). In this work the, the Corvus [opcons] workflow yields the complex dielectric function $\epsilon(\omega)$, energy loss function $L(\omega)$, complex index of refraction $\mathbf{n}(\omega)$, absorption coefficient $\mu(\omega)$, and reflectivity $R(\omega)$ defined as follows, in
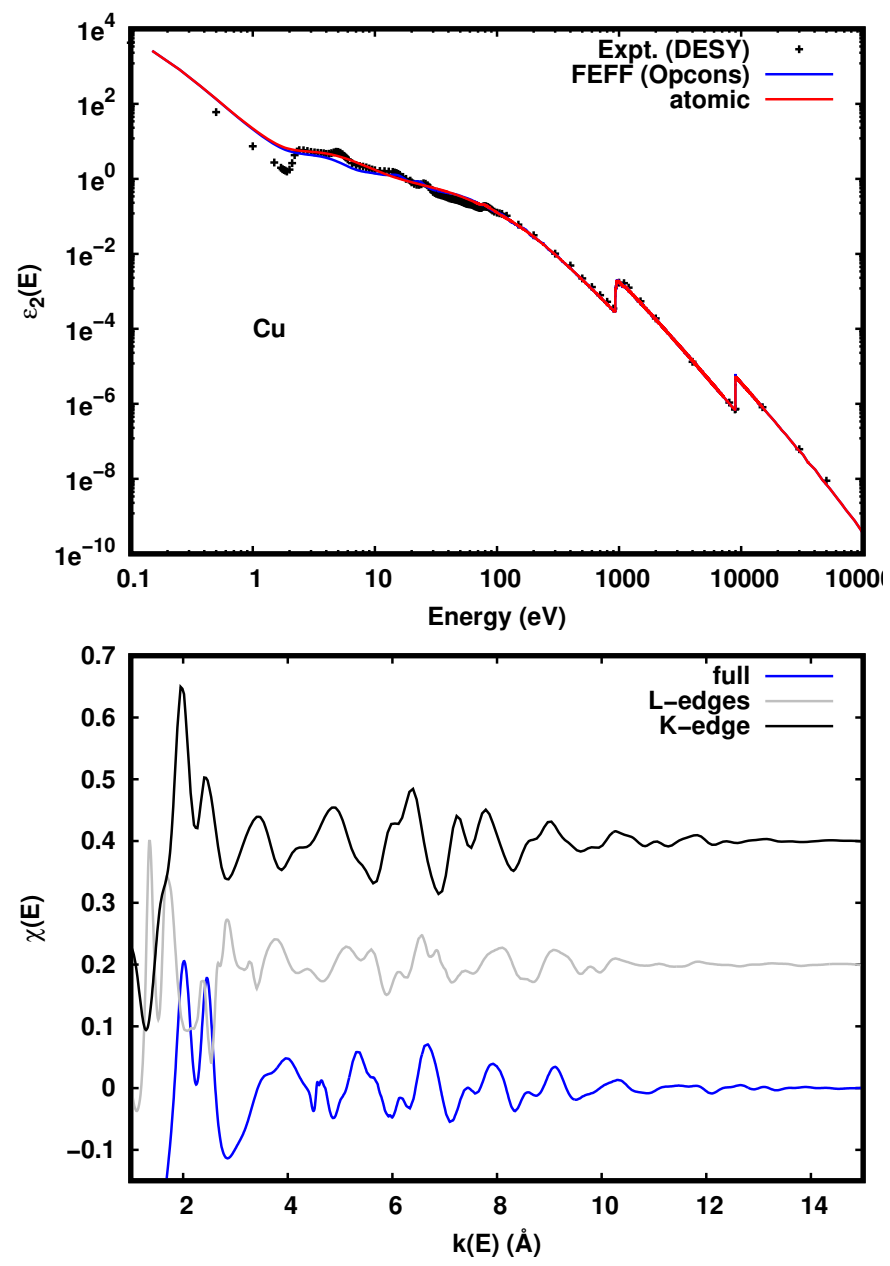

Figure 1: Top: Calculated imaginary part of the dielectric function $\epsilon_{2}$ for $\mathrm{Cu}$ (blue) along with the atomic background $\chi$ (see text) (red). Bottom: Approximate optical fine structure of the imaginary (blue) part of $\epsilon$ for $\mathrm{Cu}$ vs $k=\sqrt{2 E}$, along with the same fine-structure defined with energies $E$ relative to the $\mathrm{K}$ - and L-edges. Note the similarities between the fine structure found in the optical and that of the K-edge. Note also that the fine structure becomes negligible for large $k$ above any edges or outside the optical range.

terms of $\epsilon_{2}(\omega)$

$$
\begin{aligned}
& \epsilon_{1}(\omega)=1+\frac{2}{\pi} \mathcal{P} \int_{0}^{\infty} d \omega^{\prime} \frac{\omega^{\prime} \epsilon_{2}\left(\omega^{\prime}\right)}{\omega^{2}-\omega^{\prime 2}} \\
& L(\omega)=-\operatorname{Im}\left[\epsilon^{-1}(\omega)\right]=\frac{\epsilon_{2}(\omega)}{\epsilon_{1}(\omega)^{2}+\epsilon_{2}(\omega)^{2}} \\
& \mathbf{n}(\omega)=n(\omega)+i \kappa(\omega)=\epsilon(\omega)^{1 / 2} \\
& \mu(\omega)=2 \frac{\omega}{c} \kappa(\omega) \\
& R(\omega)=\frac{[n(\omega)-1]^{2}+\kappa(\omega)^{2}}{[n(\omega)+1]^{2}+\kappa(\omega)^{2}}
\end{aligned}
$$

where $\mathcal{P}$ denotes the principal part of the integral. Note that at high energies dielectric response is weak, and $\epsilon_{2}(\omega) \approx L(\omega) \approx 2 \kappa(\omega) \approx \mu(\omega)(c / \omega)$. Sum-rules for the dielectric properties provide a qualitative check on the reliability of the results. 25] Related properties, such as the local atomic polarizability $\alpha(\omega)=(\epsilon(\omega)-$ 


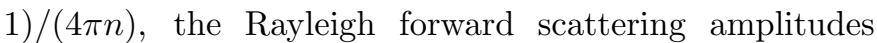
$f(\omega)=\omega \alpha(\omega) / r_{0} c^{2}$ (where $r_{0}=e^{2} / m c^{2}$ is the classical radius of the electron, $c$ is the speed of light and $n=N / V$ is the atomic number density), electron energy loss spectra (EELS), inelastic mean-free paths, Hamaker constants $\epsilon(i \omega)$, etc., can also be determined, [20] but are not yet implemented in the present Corvus [opcons] workflow. For simplicity here and below we use atomic units $e=\hbar=m=1$ throughout this paper unless otherwise needed for clarity.

The treatment of excitations from the core- and valencelevels generally requires different considerations, so it is convenient to separate the calculations as

$$
\epsilon_{2}=\epsilon_{2}^{\text {core }}+\epsilon_{2}^{\mathrm{val}},
$$

where $\epsilon_{2}^{\text {core }}$ includes the contribution from all levels below a fixed core-valence separation energy $E_{c v}$, which in FEFF10 is set to $-40 \mathrm{eV}$. Although this separation is approximate due to many-body effects, the contributions from deep core levels (and to a reasonable approximation, semi-core states) respond nearly independently from the valence.

\subsection{Core level contributions}

In this paper calculations of the optical constants are carried out using the real-space Green's function code FEFF10 and an updated version of the approach described by Prange et al. [20] FEFF uses the same realspace Green's function formalism to calculate a variety of spectroscopies and related quantities including XAS, XES, RIXS, EELS, and Compton. Thus the calculations of the optical constants use essentially the same ingredients as those for the well-established calculations of XAS, greatly simplifying the workflow design.

Briefly, the RSGF approach in FEFF is used to calculate the contribution to $\epsilon_{2}(\omega)$ from each occupied level $i$ (i.e., absorption edge) at each unique site in the system $a$. For simplicity, the site index will be usually suppressed below. The core level contribution to $\epsilon_{2}(\omega)$ from a given site $a$ consists of a sum of contributions from occupied core levels $|i\rangle\left[20\right.$ for $\varepsilon_{i}<E_{c v}$ given by

$$
\epsilon_{2}^{\text {core }}=\sum_{i} \frac{4 \pi}{\omega} \operatorname{Im}\left[\left\langle i\left|\hat{d}^{\dagger} G\left(\omega+\varepsilon_{i}\right) \hat{d}\right| i\right\rangle\right] \theta\left(\omega-\varepsilon_{F}+\varepsilon_{i}\right),
$$

where $|i\rangle$ is the core-orbital of interest, $\varepsilon_{i}$ its binding energy, $\hat{d}$ is the transition operator, which in most cases is dipole dominated, and $G(\omega)$ is the one-electron Green's function. Finally, $\theta\left(\omega-\varepsilon_{F}+\varepsilon_{i}\right)$ is a unit step function that turns on when the photon energy $\omega$ is sufficient to excite the core-electron above the Fermi energy $\varepsilon_{F}$. If the interaction between the core- and photoelectron levels is ignored, this level of approximation is equivalent to the RPA. However, in this work we use the final-state rule approximation to the core hole interaction, in which the photoelectron Green's function is calculated in the presence of a self-consistently screened core-hole.

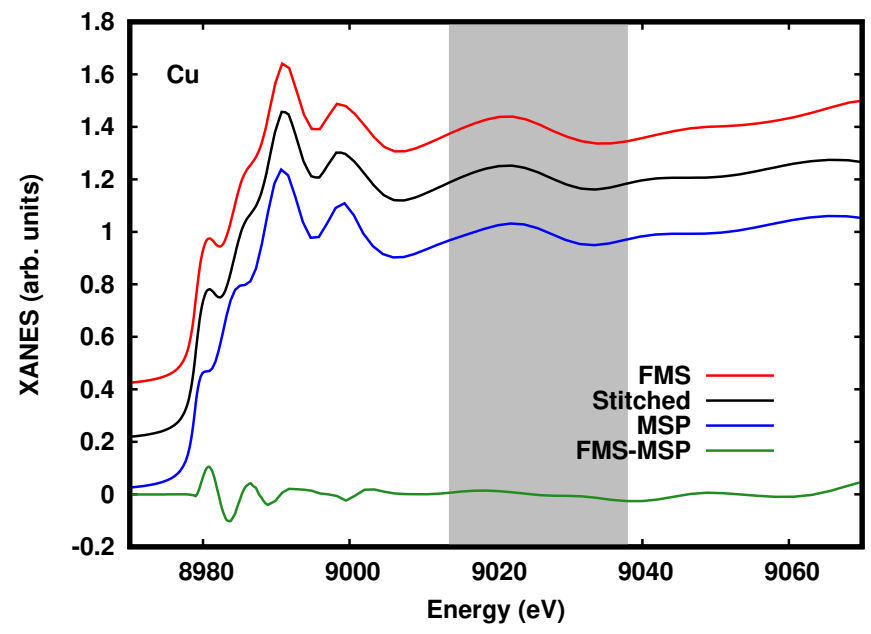

Figure 2: Cu K-edge spectrum obtained using the stitching algorithm (black). For comparison, the curves calculated using FMS (red) and MSP (blue) are also shown, shifted above and below the stitched curve, respectively. The green curve is the difference between the FMS and MSP calculated results, and the shaded area shows the region where the stitching algorithm is applied.

One of the advantages of the RSGF approach is the separation of the propagator $G(\omega)$ into contributions from the central absorber at a given site and single or multiple-scattering contributions from neighboring atoms, i.e., $G(\omega)=G^{\mathrm{abs}}(\omega)+G^{\mathrm{sc}}(\omega)$. As a result the dielectric properties exhibit fine structure analogous to that in XAFS, i.e.,

$$
\epsilon_{2}=\sum_{i} \epsilon_{2 i}^{\text {atomic }}\left[1+\chi_{i}(\omega)\right],
$$

which we dub "optical fine structure" (OFS). Typically $\chi_{i}(\omega)$ is of order a few percent within a range of order 100 $\mathrm{eV}$ above each edge; however, $\chi_{i}(\omega)$ can be large and of order unity in the near-edge regime, within about $10 \mathrm{eV}$ or so of a given edge. Thus the physical interpretation of the OFS is similar to that for EXAFS and XANES, reflecting the local geometrical structure in the vicinity of an absorbing atom. These observations also explain why a local, atomic approximation that neglects the fine structure from neighbor scattering can be a reasonable approximation, accurate to within a few percent for the core contributions to the optical constants, except close to an absorption edge. In order to show the fine structure over the entire energy range of our calculations, it is convenient to calculate the fine-structure defined as $\chi_{2}=\left(\epsilon_{2}-\epsilon_{2}^{\text {atomic }}\right) / \epsilon_{2}^{\text {atomic }}$, which is shown for $\mathrm{Cu}$ in Fig. 1 (bottom), together with the full and atomic approximation to $\epsilon_{2}$ (top). A corresponding approximation can also be made for any of the other optical constants. When $\epsilon_{1}^{\text {core }} \approx 1$, the fine structure in the loss function $L(\omega)$, absorption $\mu$ and imaginary part of the index of refraction $\kappa$ are similar to that in $\epsilon_{2}$. The fine structure in $\epsilon_{1}$ is similar, although phase shifted by $90^{\circ}$.

In order to obtain optical constants over a broad energy range from the UV to hard x-rays, two FEFF cal- 
culations must be performed for each edge (i.e. each occupied atomic level $i$ ) and each unique atomic site $a$ in the presence of the corresponding core-hole: 1) In the near edge regime within about $50 \mathrm{eV}$ of a given edge full-multiple-scattering (FMS) calculations must be performed, and 2) at higher energies (the EXAFS regime), the multiple-scattering path expansion (MSP) is used. Once these calculations are performed, the results are interpolated onto a common energy grid and stitched together to yield the spectrum $\epsilon_{2}^{i}(\omega)$ coming from the core-state $|i\rangle$. The stitching algorithm used is: 20

$$
\begin{array}{rlr}
\epsilon_{2}^{i}(k)= & \epsilon_{2}^{\mathrm{FMS}, i}(k) ; & k<k_{0}, \\
= & \cos ^{2}\left(\frac{k-k_{0}}{k_{1}-k_{0}}\right) \epsilon_{2}^{\mathrm{FMS}, i}(k) & \\
& +\sin ^{2}\left(\frac{k-k_{0}}{k_{1}-k_{0}}\right) \epsilon_{2}^{\mathrm{MSP}, i}(k) ; & k_{0} \leq k \leq k_{1}, \\
= & \epsilon_{2}^{\mathrm{MSP}, i}(k) ; & k>k_{1}
\end{array}
$$

where $k_{0}=3 \AA^{-1}, k_{1}=4 \AA^{-1}$, and $k=\left[2\left(E-E_{\text {edge }}\right)\right]^{1 / 2}$ is the photoelectron wave-number. This is illustrated in Fig. 2 for the K-edge spectrum of fcc $\mathrm{Cu}$.

\subsection{Valence Contributions}

The contribution from the valence levels is complicated by the extended range and continuum nature of the valence wave functions and the large density of states in the valence region. In the full RSGF approach this requires sums over multiple sites as in Eq. (32) of Ref. [20]. Moreover, systems with strong excitonic effects often require treatments beyond the RPA such as TDDFT or the Bethe-Salpeter equation. 9] Several codes are available for these advanced treatments, [10, 11, 13, 15] but they are computationally challenging for high throughput calculations. Nevertheless, neglecting these effects is often a reasonable approximation for semi-quantitative purposes. Consequently, in this work we use a local approximation at the absorption site to calculate the contribution from the valence electrons. In addition, we replace the valence wavefunctions with the Dirac-Fock atomic eigenfunctions, which allows us to write Eq. (32) of Ref. 20] as an integral over the local valence density of states

$$
\epsilon_{2}^{\mathrm{val}}(\omega)=\sum_{i a} \int_{E_{c v}}^{E_{F}} d \omega^{\prime} \tilde{\rho}_{a i}\left(\omega^{\prime}\right) \epsilon_{2}^{i a}\left(\omega-\Delta-\omega^{\prime}\right),
$$

where $\epsilon_{2}^{i a}(\omega)$ is the local atomic spectrum at a given site $a$ corresponding to atomic state $i$, and $\tilde{\rho}=\rho / N$ is the normalized, angular momentum projected density of states (LDOS) with symmetry equal to that of the state $i$, and $\Delta=E_{\text {top }}-E_{\text {edge }}$ is a shift to properly position the valence spectra, where $E_{\text {top }}$ is the energy at the top of the gap, if one exists, and is equal to the Fermi energy otherwise. As shown for the energy loss of $\mathrm{Al}_{2} \mathrm{O}_{3}$ (top) and $\mathrm{Au}$ (bottom) in Fig. 3, which provide good tests for this procedure we find that the approximation is reasonably accurate when
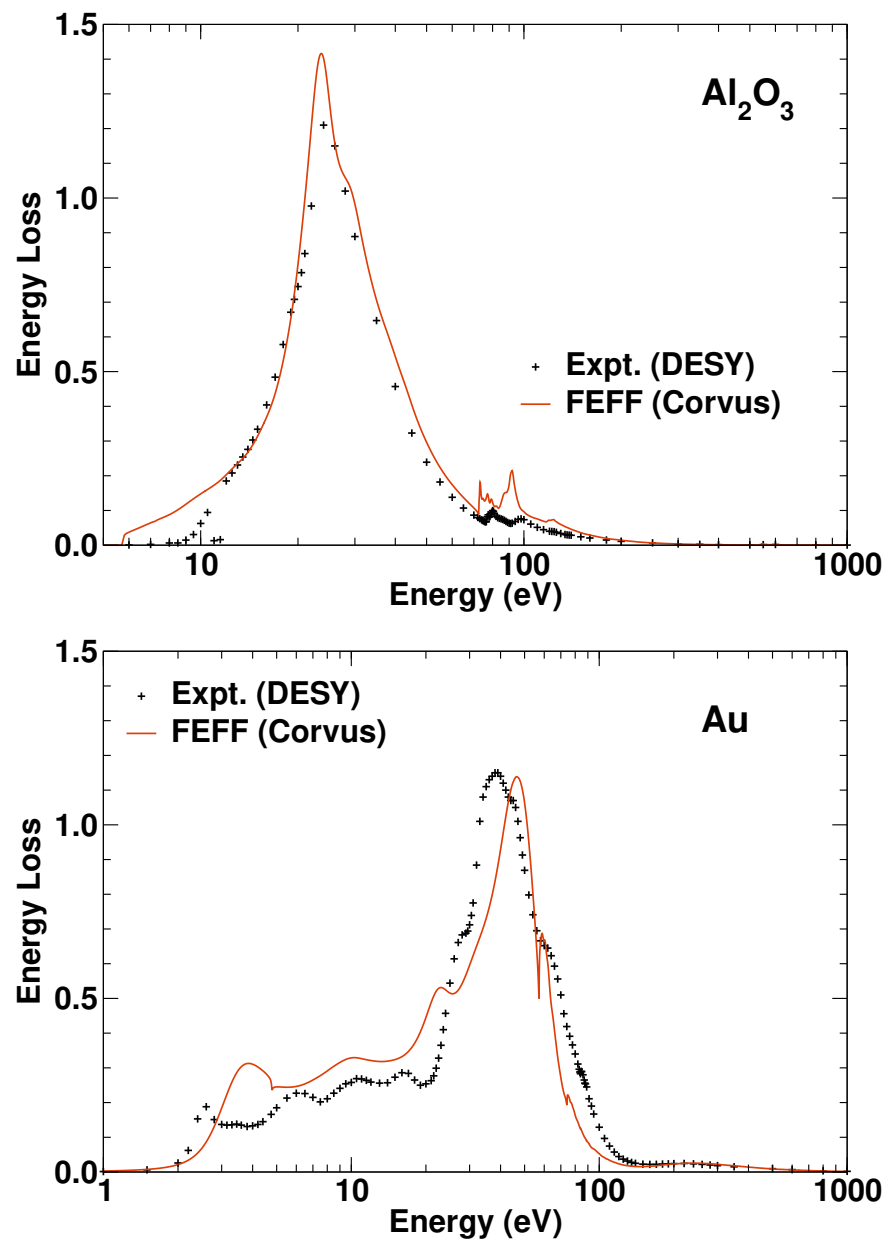

Figure 3: Comparison of the experimental [26] and calculated energy loss function for $\mathrm{Al}_{2} \mathrm{O}_{3}$ (corundum) (top) and $\mathrm{Au}$ (gold) (bottom) with the local approximation (see text).

excitonic effects are small. We have found that our local approximation in Eq. 10 is significantly better than a pure atomic approximation alone. One can also define an optical fine structure $\chi^{\mathrm{val}}$ for the valence contributions $\epsilon_{2}^{\mathrm{val}}$, although its magnitude is typically large and of order unity (see Fig. 1), as in core-level XANES, over the optical energy range. For this reason, FMS and the local projected densities of states are needed to evaluate Eq. (10). In

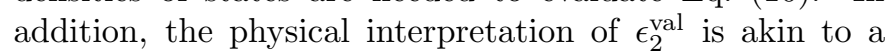
sum of terms proportional to the joint projected density of states. 20] Even so, the valence fine structure for $\mathrm{Cu}$ is rather similar to the K-edge fine structure, as shown in the lower panel of Fig. 1. This makes sense, since the $d$ - to $p$-state transitions dominate in the optical region, and the DOS is dominated by a single, relatively sharp occupied $d$-band.

Finally, the total $\epsilon_{2}(\omega)$ is obtained from a double sum over edges $i$ and unique sites in the system,

$$
\epsilon_{2}(\omega)=\sum_{i a} n_{a} \epsilon_{2}^{i a}(\omega)
$$

where $a$ denotes a unique site in the unit cell, and $n_{a}=$ 


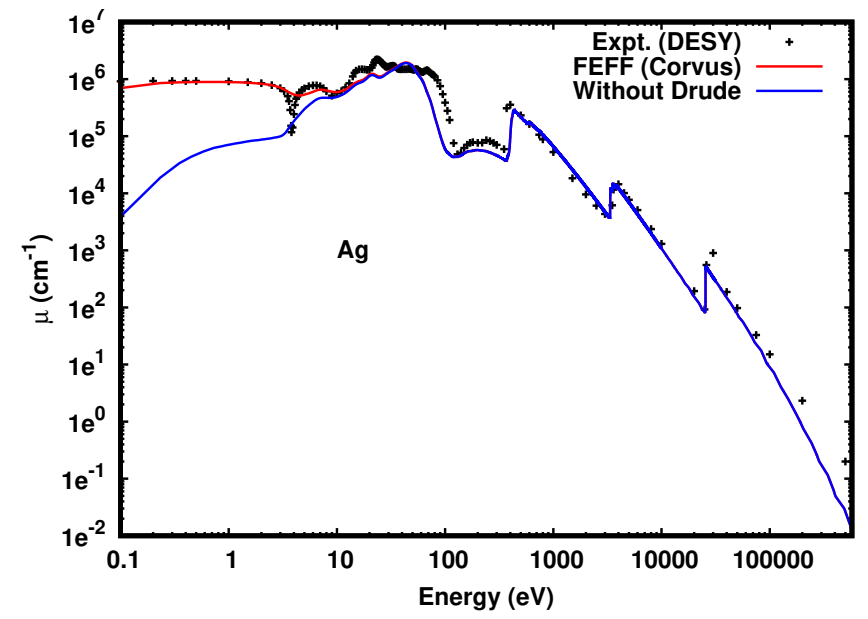

Figure 4: Comparison of the theoretical absorption $\mu$ of $\mathrm{Ag}$ with and without the Drude contribution, along with experimental results [26].

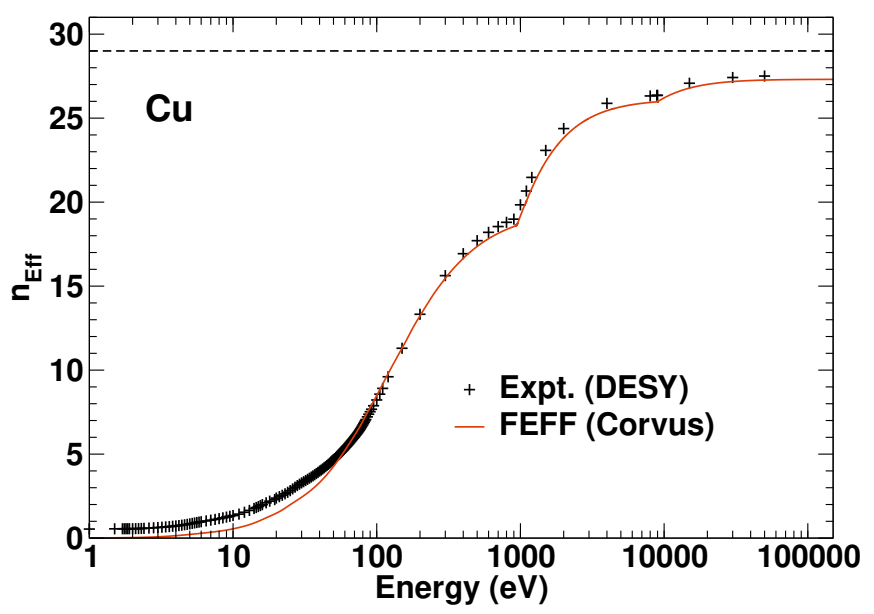

Figure 5: Comparison of the experimental 26] and theoretical $\epsilon_{2}(\omega)$ sum rule $n_{\text {eff }}(\omega)=\int_{0}^{\omega} d \omega \epsilon_{2}(\omega) / \pi$ for $\mathrm{Cu}$. The horizontal dashed line indicates the ideal theoretical limit $(Z=29)$.

$N_{a} / V$ is the corresponding number density.

\subsection{Debye-Waller factors and Drude corrections}

A number of other considerations are generally important in calculations of optical spectra over broad energy ranges. For example, effects of thermal vibrations are temperature dependent and strongly damp the fine-structure in the EXAFS regime. For the systems treated here we have approximated the multiple-scattering path dependent DW factors $\exp \left(-2 \sigma^{2} k^{2}\right)$ using the default correlated Debye model in FEFF10. The Debye temperature parameter can be estimated 27 from the bulk and shear elastic moduli data available in the MP database.

Drude corrections in metals can be added phenomenologically by an additional ad hoc contribution to the limiting low frequency behavior of $\epsilon_{2}(\omega)$ given by

$$
\epsilon_{2}^{D}=\frac{4 \pi \sigma}{\omega}=\frac{\omega_{p}^{2} \tau}{\omega}
$$

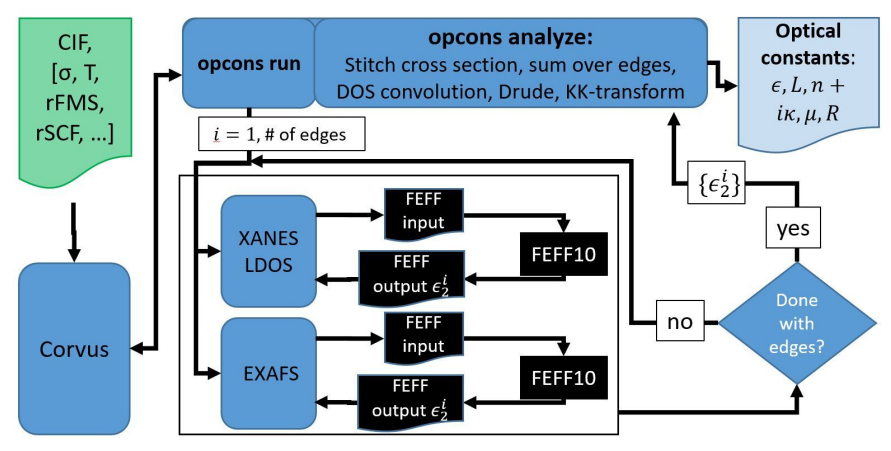

Figure 6: Schematic of the Corvus workflow for optical constants. User input is shown in light green, while code blocks associated with Corvus are shown in blue, and input/output/code associated with FEFF is shown in black. The output is shown in light blue. Note that Corvus [opcons] requires minimal input from the user (only the CIF file and a request for optical constants), although many optional parameters are available.

where $\sigma$ is the conductivity, $\omega_{p}^{2}=4 \pi n e^{2} / m, n$ is the mean conduction electron density, and $\tau$ the relaxation time, which is typically of order $0.1-4.0$ femtosecond at room temperature. 28] Fig. 4 shows a comparison of the absorption $\mu$ of $\mathrm{Ag}$ with and without the Drude contribution, along with experiment. [26]

Thermal expansion can also be treated by calculating the expansion parameter via DFT, 29] or using parameters from the literature. Additional broadening due to electron-phonon interactions, impurities, and other mechanisms can also be important. While neglected by default, these effects can be added ex post facto by a Lorentzian broadening of the spectra.

Once $\epsilon_{2}(\omega)$ is obtained by summing both core and valence contributions over all sites, the real part of the dielectric function is formed via a KK-transform, as in Eq. (1). A check on the completeness of the calculations is provided by the optical constant sum-rules, 25] as illustrated in Fig. 5.

\subsection{Corvus [opcons] workflow}

The Corvus [opcons] workflow is built on the concept of recursive workflow generation, wherein Corvus generates and runs a workflow with the target property opcons, and the optical constants workflow [opcons] in turn generates and runs a [xanes] workflow for each required FEFF10 calculation. The workflow tool is described in detail together with various examples in the Corvus reference. 24 This approach determines the input files from the data in the MP database, afterwhich all of the individual FEFF10 calculations are set up and run through the Corvus workflow manager. The Corvus [opcons] workflow then carries out the following steps: i) calculating the $\epsilon_{2}(\omega)$ for a given edge; ii) applying the stitching algorithm to combine the near-edge full multiple scattering (FMS) and multiplescattering path (MSP) calculations; iii) convolving with the density of states (Eq. 10p; iv) performing the KKtransform to obtain $\epsilon_{1} ; \mathrm{v}$ ) adding the Drude contribution; 


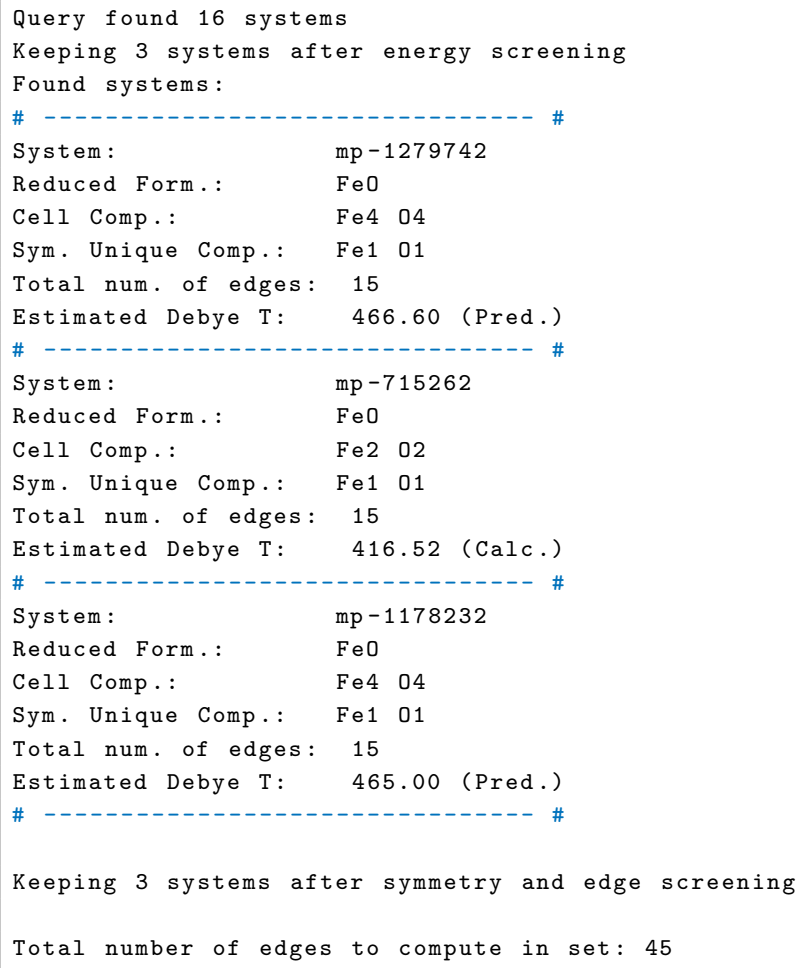

Figure 7: Typical output for the crv_mp_mk_set command showing that the MP query returned 16 possible systems, of which only 3 are left after screening for energy, symmetry, and total number of edges. Also shown are the estimated Debye temperatures, which can be calculated (e.g., from DFT data), or predicted (e.g., from machine learning data).

and vi) calculating the other optical constants following the relations in Eqs. (1). In order to facilitate automated calculations, Corvus handles the creation of FEFF10 input files from minimal user input, executes FEFF10, and translates the output. Additionally, the Corvus [opcons] workflow is designed to optimize the calculations, for example re-using output from the XANES results within the EXAFS calculations to bypass the self-consistency loop. Figure 6 shows a schematic of the Corvus [opcons] workflow for producing the optical constants.

\section{Materials Project interface tools}

\subsection{Global structure of Corvus [opcons] simulations}

MP optical constants simulations are set up in two steps: 1) The crv_mp_mk_set command reads the command line inputs, creates a request to the MP, downloads all the structures available matching the query, generates a set of materials to run and calculates the totl number of edges in the set. 2) The crv_mp_run_set command runs all the calculations required for the set, distributed in serial or parallel mode according to the resources provided. In the following section we describe their usage in detail.

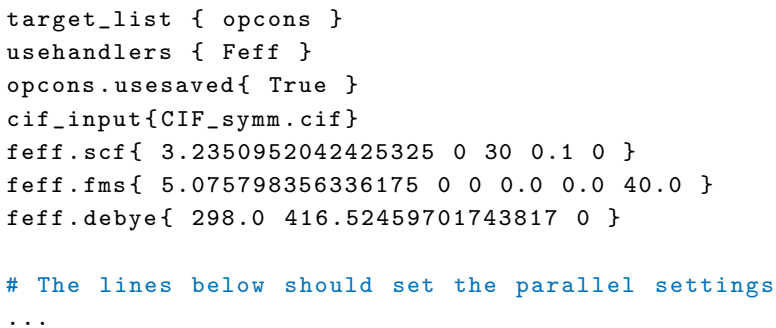

Figure 8: Typical template Corvus [opcons] input generated by the crv_mp_mk_set command. This input shows the selected Corvus target opcons, the handlers that sould be used (only FEFF is required for these types of runs), the location of the structure in CIF format, and the FEFF SCF and FMS radii. The latter are automatically generated based on the number of shells requests in the command line. Depending on the options used, the calculation can use a Correlated Debye model to add thermal broadening, using a Debye temperature parameter estimated from MP database values of the bulk and shear moduli. Given that Corvus stores the MPI command, number of processors, etc in the input, the actual input is generated at runtime by the crv_mp_run_set, where the required parameters are added at the end of the template input shown here.

\subsection{Usage: $c r v \_m p \_m k \_s e t$}

The basic input of a typical Corvus [opcons] calculation is set up using the crv_mp_mk_set command:

crv_mp_mk_set [OPTIONS] Set_Name

where "Set_Name" is a text label that will be used throughout to refer to the set of materials. This label is used to name the directory where the calculations will be done and the results stored. crv_mp_mk_set can be used to query the MP database in three different ways (see options below): 1) Query an $n$ arbitrary class of materials selected by formula, 2) automatically query some or all of the elemental solids, for which the tools have an internal database of MP-IDs, and finally, 3) an individual material with a given MP-ID. The full set of options can be obtained with "crv_mp_mk_set --h". Here we focus on some of the most computationally relevant ones.

\subsubsection{Required options}

\section{$--\mathrm{k}$ APIKEY}

API key used to interact with the MP REST interface

This option is required for the command to be able to interact with the MP database. See section 3.4.4 for details.

\subsubsection{System selection options \\ --f FORMULA}

Use chemical formula FORMULA to define a set of materials.

- -pt

Create a set for all the solid elements in the periodic table using the structure for the most stable phase in standard conditions

--ptel PT_ELEMS

Same as --pt, but only for a list of specific elements 


\section{--mpid MP-ID}

Select the individual system with Materials Project identification label MP-ID (Default: "mp-30")

These options are mutually exclusive. If none of them are present the command generates input for a test run on $\mathrm{Cu}$, a traditional choice since FEFF was first deployed.

\subsubsection{Selection fine-tuning options \\ --mxpt MXNAPT}

Maximum atomic number to use while creating the periodic table with --pt (Default: 99)

--enpct EN_PCT

Keep only EN_PCT\% of lowest energy systems (Default: $100 \%)$

\section{--nedg MXNEDGES}

Keep only systems with fewer than MXNEDGES edges (Default: 250)

These options help trim the raw selection generated by the system selection options.

\subsubsection{Auxiliary options \\ $--n S C F$ NSCF}

Number of neighbor shells to use in SCF (Default: 1)

--nFMS NFMS

Number of neighbor shells to use in FMS (Default: 2)

\section{--symprec SYM_PREC}

Symmetry precision (in $\AA$ ) used by symmetrizer (Default: $0.01 \AA$ )

\section{$--d w$}

Use the correlated Debye thermal broadening model in the optical constants calculation

\section{--temp TEMPERATURE}

Temperature (in K) used for correlated Debye thermal broadening (Default: 298K)

These options control the setup of the optical constants calculations by defining how many shells to use in the SCF and FMS steps, as well as the precision of the symmetrization procedure used to reduce the number of unique sites. The shell selection is performed using the Jenks natural breaks classification method 30, as implemented in the jenkspy Python module. 31] Additionally, vibrational broadening can also be controlled by requesting that a correlated Debye model 32 be used.

\subsubsection{Calculation control options}

\section{$--\mathrm{dr}$}

Dry run, collect and display information without actually creating a set

$$
--\mathrm{v}
$$

Make the command output more verbose
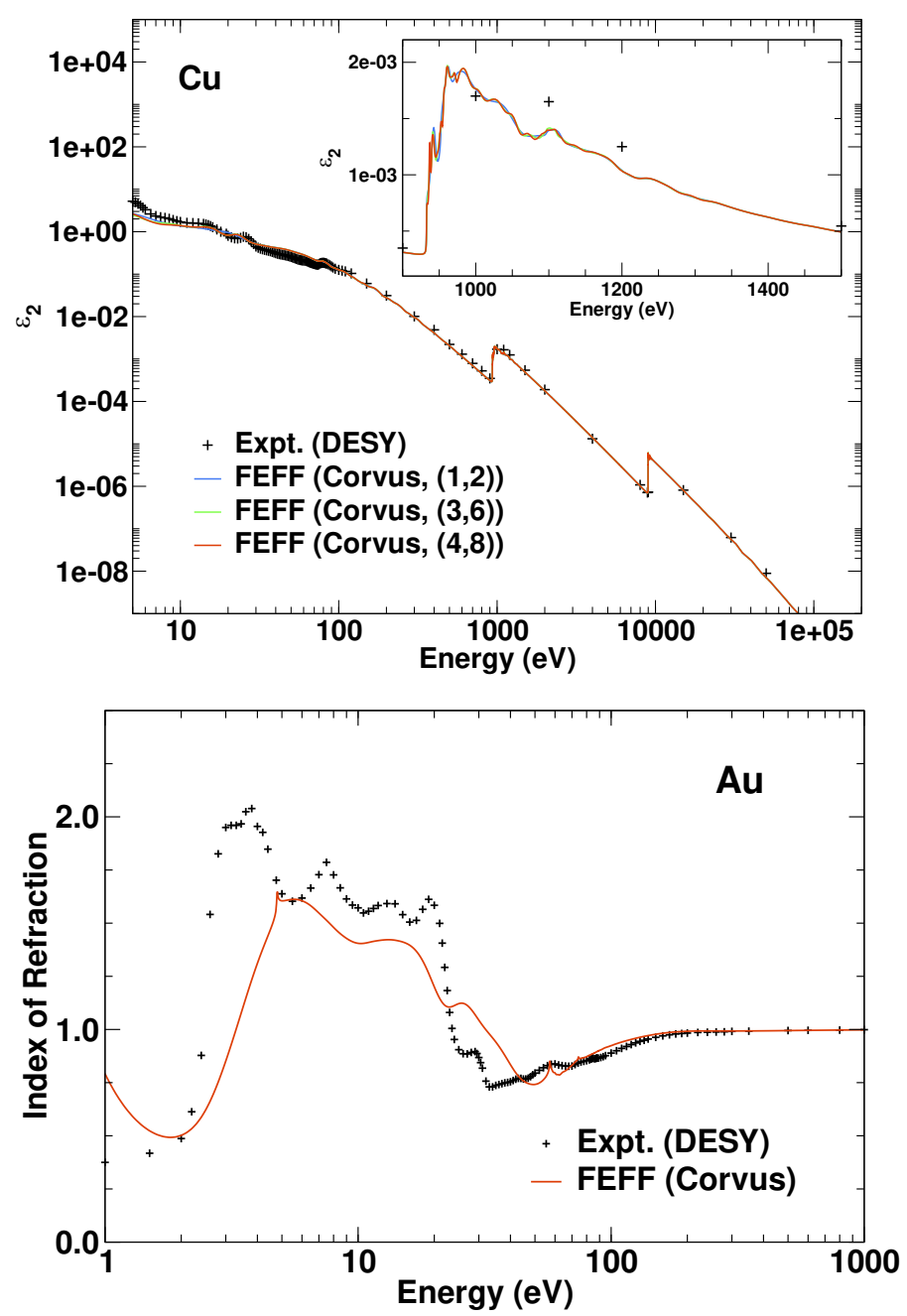

Figure 9: Comparison of the experimental 26] and theoretical $\epsilon_{2}$ for $\mathrm{Cu}$ as a function of FEFF Corvus (nSCF,nFMS) shells to explore convergence (top) and (bottom) index of refraction for $\mathrm{Au}$ in the valence region (bottom).

\subsubsection{Examples}

A typical invocation of crv_mp_mk_set is as follows crv_mp_mk_set --k [APIKEY] --f "FeO" --enpct 20 --nedg 250 --nSCF 2 --nFMS 4 --v --dw --temp 298.0 IronOxides

creates a set of 3 systems (Figure 7) after screening for energy, symmetry and total number of edges. The command also requests that the Debye temperature be estimated, and that the calculation be done at room temperature. Inside the set directory, the structures are organized by reduced formula and MP-ID. A typical Corvus [opcons] directory created with crv_mp_mk_set contains, for each material, a template Corvus [opcons] input (Fig. 8) and a few auxiliary files with structural information. A set for the Li, Be and B elemental solids can be generated using:

crv_mp_mk_set --k [APIKEY] --v --ptel 'Li, Be, B' LiBeB

while a single compound (one of the forms of $\mathrm{Mg}_{3} \mathrm{~N}$, in this case) can be set up with: 


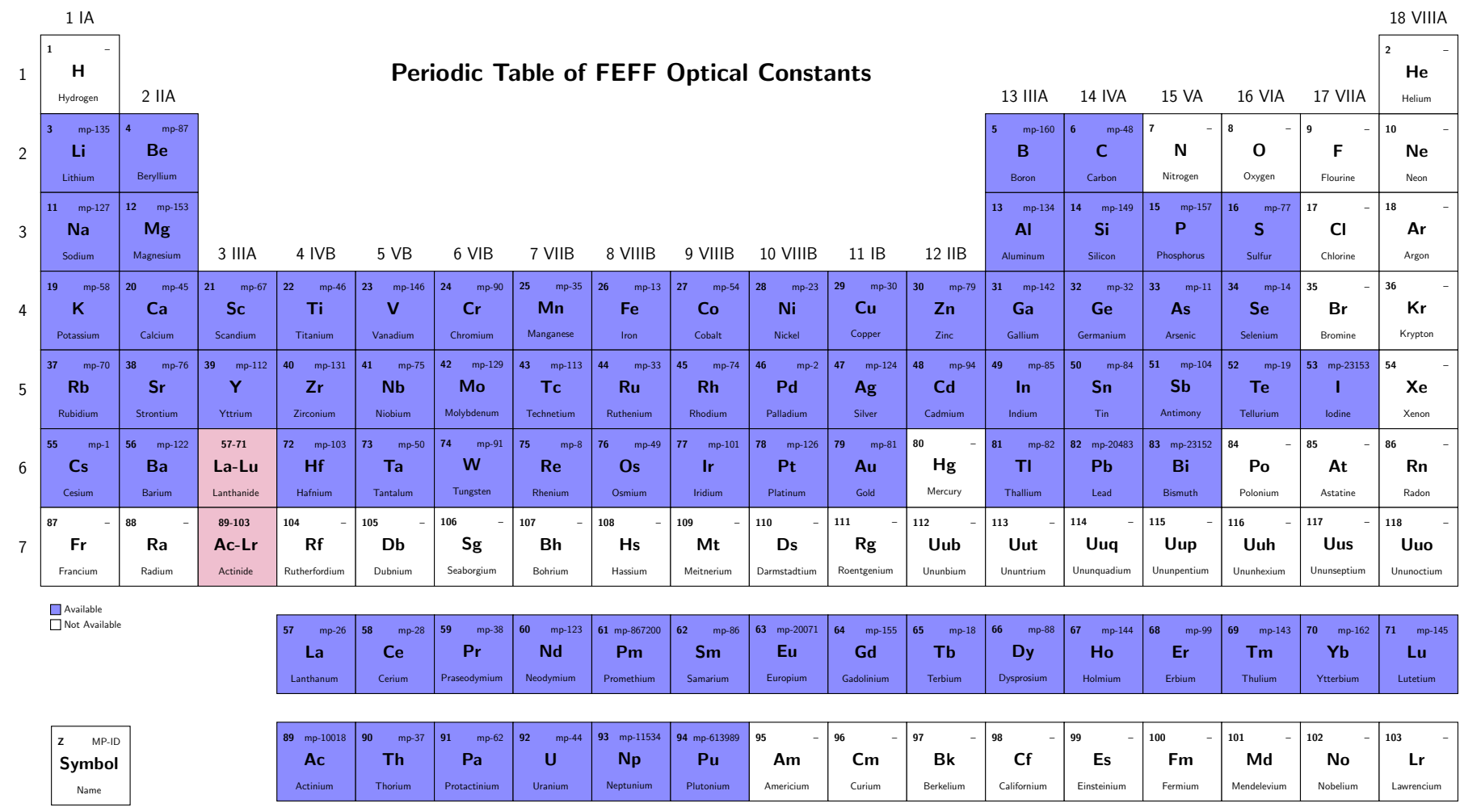

Figure 10: Periodic table of FEFF optical constants currently available. The index on the top-right corner of each element indicates the MP-ID of the structure used in the calculations. A dynamic version of this table can be found at http://feff.phys.washington.edu/ optical-constants/,33 including all optical constants data as well as the input files used to generate it.

\author{
crv_mp_mk_set --k [APIKEY] --v --mpid \\ $\mathrm{mp}-1185783$
}

More examples can be found in Ref. [34].

\subsection{Usage: $c r v \_m p \_r u n \_s e t$}

After a set is created, the actual optical constants can be computed with the crv_mp_run_set command:

crv_mp_run_set [OPTIONS] Set_Name

where "Set_Name" is again the label for the set to run. As for "crv_mp_mk_set", the full set of options can be obtained with "crv_mp_run_set --h", but here we focus on some of the most computationally relevant ones.

\subsubsection{Options to control the number of processors --np NP_TOT}

Total number of processors to be used in the run (Default: 1)

\section{--ppn PPN}

Number of processors per node (Default: 1)

\section{$--\mathrm{nn}$ NNODES}

Number of nodes with PPN processors per node to be used in the run (Default: 1)

If more than one of these options are specified, the command will choose max (NP_TOT, PPN*NNODES) as the total number of processors to use.

\subsubsection{Options to estimate computational cost $--t$}

Estimate total runtime up to NP_TOT processors

\section{--sf SER_FRAC}

Fraction of serial code to estimate parallel runtimes (Default: 0.5$)$

If the "--t" runtime option is present, instead of running the set, crv_mp_run_set prints out estimates of the cost of the calculation from 1 to NP_TOT processors relative to the cost of a single edge calculations performed in serial mode.

\subsubsection{Auxiliary options \\ $--\mathrm{v}$}

Generate verbose output (timings, etc)

$--d r$

Dry run: Do everything but actually run

\subsubsection{Examples}

A typical instance of crv_mp_run_set to run the set IronOxides created in the previous example (Fig. 7) is as follows:

$$
\begin{aligned}
& \text { crv_mp_run_set --ppn } 64-\mathrm{nn} 45--\mathrm{v} \text { Iron0xides } \\
& \text { crv_mp_run_set -np } 2880--\mathrm{v} \text { Iron0xides }
\end{aligned}
$$
or

Given that the set contains 45 edges, for maximum efficiency we assign one node with 64 processors to each 
edge, or a total of 2880 processors, thus effectively running all materials simultaneously. FEFF10 then computes each individual edge parallelizing over the energy grid with 64 processors.

\subsection{Requirements}

\subsubsection{Code distribution}

Both Corvus 35 and FEFF10 36 are open source and are available on GitHub. Detailed installation instructions can be found in Ref. 34.

\subsubsection{Corvus requirements}

The Corvus workflow manager is implemented in Python 3. In addition, it requires the Numpy [37] and Scipy [38] packages, as well as a few other packages depending on the required functionality. The Corvus workflows that are possible will be determined by the specific external software packages installed on the system. In this case, the optical constants WF only requires the appropriate version of FEFF10.

\subsubsection{FEFF10 requirements}

To run efficiently, a parallel execution is important. Thus, the FEFF code requires a Fortran90 compiler with some flavor of MPI-2 for compilation. FEFF has been tested with a wide variety of compilers and MPI-2 implementations.

\subsubsection{Corvus [opcons] specific requirements}

The crv_mp_mk_set and crv_mp_run_set tools are also implemented in Python 3 and, in addition to the Corvus requirements, they need the pymatgen package. 39] In order to use the MPRester method in pymatgen to access the Materials Project database directly, users need to have an MP account and a valid API key. For the work presented in this paper, we used pymatgen v2022.0.9, and MP v2019.05.

\subsubsection{Performance Analysis}

The CPU time for a given material scales linearly with the number of edges and unique atom positions in a unit cell and, depending on the SCF and FMS settings, typically takes between a few minutes and $1 / 2 \mathrm{hr}$. Thus, when the available number of processors is equal or greater than the total number of edges, linear parallelization is simply achieved by assigning a processor or more to each edge, up to about $32-64$ processors. More processors per edge becomes less efficient at the level of FEFF calculations. In other cases, and for high-throughput runs for many different materials, the wall-clock time depends on how the set is structured (e.g., how many edges per material in the set) and how the calculations are distributed in the workflow. In that case, there are no simple rules to determine the optimal parallel distribution. To help with this issue, the crv_mp_run_set tool includes an option ("--t") to estimate how well a given system of interest will parallelize for a certain range of number of processors ("--np") relative to the fully serial calculation.

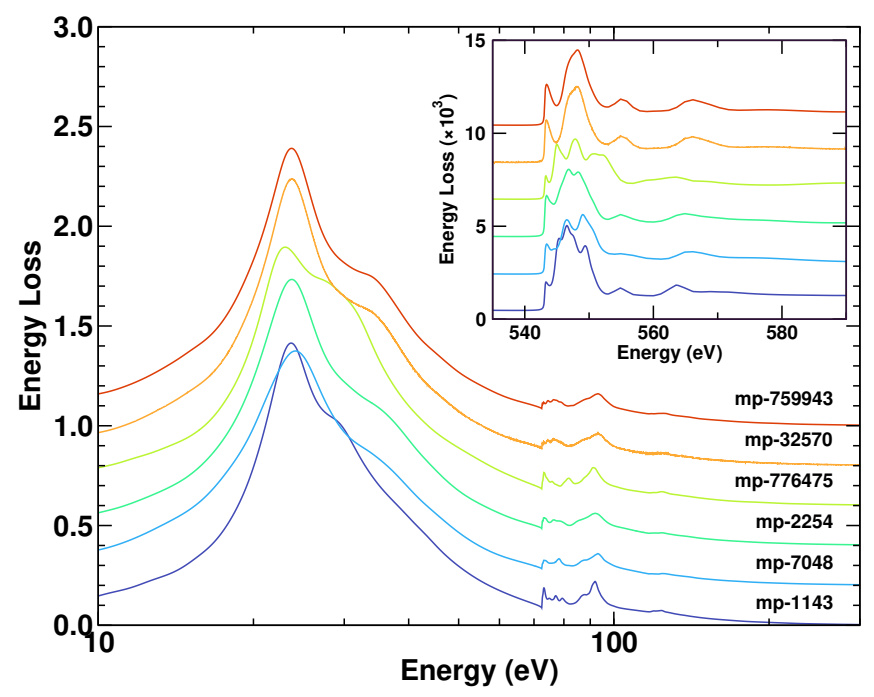

Figure 11: Loss function for several $\mathrm{Al}_{2} \mathrm{O}_{3}$ oxides listed in the MP.

\section{Example Applications}

\subsection{Optical constants of the elements}

As an illustration, we have carried out calculations for the most stable structure (at room temperature and nominally $1 \mathrm{~atm}$ ) of each of the elemental solids listed in the MP with data imported for a given MP-ID. Some of the most important factors limiting the quality of the results are the SCF and FMS radii used in the simulations. The results can be obtained online at http://feff .phys. washington.edu/optical-constants/33. Fig. 9 (top) shows a comparison of the results using an increasing number of near-neighbor shells for $\epsilon_{2}$ of copper. We find that 3 and 6 shells in the SCF and FMS modules, respectively, produce converged results above $10-20 \mathrm{eV}$.

Overall, the results are in reasonable agreement with experimental tabulations, 26] and illustrate that, except close to an edge or in the optical region, $\epsilon_{2}$ can be approximated reasonably by the atomic approximation, ignoring the fine structure. For example, Fig. 9 shows a comparison to experiment [26] of $\mathrm{Cu} \epsilon_{2}$ (top) and real index of refraction $\mathrm{Au}$ (bottom) computed with converged SCF and FMS radii and including the density of states convolution in Eq. 10. These results show that Eq. 10 is only semi-quantitative in the visible range. Fig. 10 shows a periodic table of the elements and associated MPIDs that are currently available and can be freely viewed and downloaded at http://feff .phys.washington.edu/ optical-constants/,33.

\subsection{Optical constants of the $\mathrm{Al}_{2} \mathrm{O}_{3}$ materials in the $\mathrm{MP}$}

In addition to the elemental solids, we have also computed several aluminum oxides. For example, Fig. 3 shows 
a comparison to experiment 26] of the energy loss for the full energy range for the corundum form of $\mathrm{Al}_{2} \mathrm{O}_{3}$. In order to demonstrate the set capabilities of the Corvus [opcons] workflow, we calculated results for a sub-set of those listed in the MP with formula $\mathrm{Al}_{2} \mathrm{O}_{3}$. The results for the loss function up to the Al L-edges are shown in the main panel of Fig. 11, while the inset highlights the O Kedge region. The differences in the near edge structure, as well as those in the optical reflect the different symmetries present in the system. For example, there is a mixture of $\mathrm{Al}$ sites in octahedral and tetrahedral symmetry in the first two materials, which are both monoclinic, while the last material is trigonal, and contains only $\mathrm{Al}$ in octahedral symmetry. Similarly, the oxygen sites in the first two systems are a mixture of sites in tetrahedral and trigonal symmetry. These changes in symmetry lead to differences in electronic structure, which are directly related to the features seen in the near edge spectra.

\section{Conclusions}

We have presented an optical constants ("opcons") workflow built with the Python-based Corvus workflow manager for high-throughput simulations of full spectrum optical constants from the UV-VIS to hard x-ray wavelengths for materials defined in the MP data-base. The calculations are based on the efficient RSGF code FEFF10 and an approximate treatment of the valence contributions using structural data from the MP database specified by a unique MP materials ID. As for the XAS, the results can be represented in terms of an atomic-like background and an optical fine-structure (OFS) analogous to EXAFS. Except close to an edge where the fine-structure is substantial, the background dominates and can be calculated efficiently over the full spectrum. The fine-structure is edge-specific and as in EXAFS, reflects the structure of the environment of a given absorber in the material. The calculations are set up automatically using Corvus auxiliary tools, and the workflow parallelizes the FEFF computations over edges and sites with an optimal distribution of computational resources. Representative results show that the workflow produces accurate optical properties over a broad range of energies. Although this algorithm includes mean-free paths and Debye-Waller factors, multiplet splitting, excitonic effects, and long-range contributions to the valence spectra are currently neglected. Consequently, the present results may only be semi-quantitative in the UVVIS regime.

\section{Data availability}

We have computed the optical properties for all elemental solids in the periodic table for which structures are available in the Materials Project database, using the approach in this work. The data, including input files and structure, are freely available online at http://feff.phys.washington.edu/ optical-constants/. Complete installation and testing instructions as well as examples for the Corvus [opcons] workflow, its associated tools, and the FEFF10 code are available at http://feff.phys.washington. edu/optical-constants/installation.html.

\section{Acknowledgments}

We thank C. Draxl, S. Dwaraknath, L. Hung, L. Reining, and J. Vinson for comments and suggestions. This work was supported primarily by the US Department of Energy, Office of Basic Energy Sciences, Division of Materials Sciences and Engineering, under Contract No. DEAC02-76SF00515, specifically through the Theory Institute for Materials and Energies Spectroscopies (TIMES) program (JJR, JJK, FDV, and CDP), with computational support from NERSC, a DOE Office of Science User Facility, under Contract No. DE-AC02-05CH11231. R. X. Yang and K. A. Persson acknowledge support by the US Department of Energy, Office of Science, Office of Basic Energy Sciences, Materials Sciences and Engineering Division under Contract No. DE-AC02-05-CH11231 (Materials Project program KC23MP).

\section{References}

[1] E. D. Palik, Handbook of Optical Constants of Solids, Academic Press, Orlando, 1985.

[2] S. Adachi, Handbook On Optical Constants Of Metals, The: In Tables And Figures, World Scientific, 2012.

[3] B. L. Henke, E. M. Gullikson, J. C. Davis, X-ray interactions: photoabsorption, scattering, transmission, and reflection at $\mathrm{E}=50-30000 \mathrm{eV}, \mathrm{Z}=1-92$, Atom. Data Nucl. Data Tables 54 (1993) 181.

[4] C. T. Chantler, Detailed tabulation of atomic form factors, photoelectric absorption and scattering cross section, and mass attenuation coefficients in the vicinity of absorption edges in the soft $\mathrm{x}$-ray $(\mathrm{Z}=30-36, \mathrm{Z}=60-89, \mathrm{E}=0.1 \mathrm{keV}-10 \mathrm{keV})$, addressing convergence issues of earlier work, J. Phys. Chem. Ref. Data 29 (2000) 597.

[5] P. Nozières, D. Pines, Electron interaction in solids. general formulation, Phys. Rev. 109 (3) (1958) 741-761.

[6] S. L. Adler, Quantum theory of the dielectric constant in real solids, Phys. Rev. 126 (2) (1962) 413-420.

[7] N. Wiser, Dielectric constant with local field effects included, Phys. Rev. 129 (1) (1963) 62-69.

[8] G. Onida, L. Reining, A. Rubio, Electronic excitations: densityfunctional versus many-body green's-function approaches, Rev. Mod. Phys. 74 (2) (2002) 601-659.

[9] R. M. Martin, L. Reining, D. M. Ceperley, Interacting electrons, Cambridge University Press, 2016.

[10] H. M. Lawler, J. J. Rehr, F. Vila, S. D. Dalosto, E. L. Shirley, Z. H. Levine, Optical to UV spectra and birefringence of $\mathrm{SiO}_{2}$ and $\mathrm{TiO}_{2}$ : First-principles calculations with excitonic effects, Phys. Rev. B 78 (20) (2008) 205108.

[11] J. Vinson, J. J. Rehr, J. J. Kas, E. L. Shirley, Bethe-Salpeter equation calculations of core excitation spectra, Phys. Rev. B 83 (2011) 115106.

[12] The exc code, https://etsf.polytechnique.fr/exc/

[13] A. Gulans, S. Kontur, C. Meisenbichler, D. Nabok, P. Pavone, S. Rigamonti, S. Sagmeister, U. Werner, C. Draxl, exciting: a full-potential all-electron package implementing densityfunctional theory and many-body perturbation theory, J. Phys. Condens. Matter 26 (2014) 363202. 
[14] N. Tancogne-Dejean, M. J. T. Oliveira, X. Andrade, H. Appel, C. H. Borca, G. Le Breton, F. Buchholz, A. Castro, S. Corni, A. A. Correa, U. De Giovannini, A. Delgado, F. G. Eich, J. Flick, G. Gil, A. Gomez, N. Helbig, H. Hübener, R. Jestädt, J. Jornet-Somoza, A. H. Larsen, I. V. Lebedeva, M. Lüders, M. A. L. Marques, S. T. Ohlmann, S. Pipolo, M. Rampp, C. A. Rozzi, D. A. Strubbe, S. A. Sato, C. Schäfer, I. Theophilou, A. Welden, A. Rubio, Octopus, a computational framework for exploring light-driven phenomena and quantum dynamics in extended and finite systems, J. Chem. Phys. 152 (2020) 124119.

[15] Y. Takimoto, F. D. Vila, J. J. Rehr, Real-time time-dependent density functional theory approach for frequency-dependent nonlinear optical response in photonic molecules, J. Chem. Phys. 127 (2007) 154114.

[16] C. Ambrosch-Draxl, J. O. Sofo, Linear optical properties of solids within the full-potential linearized augmented planewave method, Comput. Phys. Commun. 175 (1) (2006) 1-14.

[17] A. Marini, C. Hogan, M. Grüning, D. Varsano, yambo: An ab initio tool for excited state calculations, Comput. Phys. Commun. 180 (2009) 1392-1403.

[18] W. S. M. Werner, K. Glantschnig, C. Ambrosch-Draxl, Optical constants and inelastic electron-scattering data for 17 elemental metals, J. Phys. Chem. Ref. Data 38 (2009) 1013-1092.

[19] E. G. Maksimov, I. I. Mazin, S. N. Rashkeev, Y. A. Uspenski, First-principles calculations of the optical properties of metals, J. Phys. F Met. Phys. 18 (1988) 833-849.

[20] M. P. Prange, J. J. Rehr, G. Rivas, J. J. Kas, J. W. Lawson, Real space calculation of optical constants from optical to x-ray frequencies, Phys. Rev. B 80 (2009) 155110.

[21] C. Zheng, K. Mathew, C. Chen, Y. Chen, H. Tang, A. Dozier, J. J. Kas, F. D. Vila, J. J. Rehr, L. F. Piper, et al., Automated generation and ensemble-learned matching of x-ray absorption spectra, Npj Comput. Mater. 4 (2018) 1-9.

[22] Y. Chen, C. Chen, C. Zheng, S. Dwarknath, M. K. Horton, J. Cabana, J. J. Rehr, J. Vinson, A. K. Dozier, J. J. Kas, K. A. Persson, S. P. Ong, Database of ab initio L-edge x-ray absorption near edge structure, Sci. Data, in press (2021).

[23] S. B. Torrisi, M. R. Carbone, B. A. Rohr, J. H. Montoya, Y. Ha, J. Yano, S. K. Suram, L. Hung, Random forest machine learning models for interpretable x-ray absorption near-edge structure spectrum-property relationships, npj Computational Materials 6 (1) (2020) 1-11.

[24] S. M. Story, F. D. Vila, J. J. Kas, K. B. Raniga, C. D. Pemmaraju, J. J. Rehr, Corvus: a framework for interfacing scientific software for spectroscopic and materials science applications, J Synchrotron Radiat 26 (2019) 1694-1704.

[25] M. Altarelli, D. L. Dexter, H. M. Nussenzveig, D. Y. Smith, Superconvergence and sum rules for the optical constants, Phys. Rev. B 6 (12) (1972) 4502-4509.

[26] H. Hageman, W. Gudat, C. Kunz, Optical constants from the far infrared to the x-ray region: $\mathrm{Mg}, \mathrm{Al}, \mathrm{Cu}, \mathrm{Ag}, \mathrm{Au}, \mathrm{Bi}, \mathrm{C}$, and $\mathrm{Al}_{2} \mathrm{O}_{3}$, Tech. rep., DESY (1974).

[27] O. L. Anderson, A simplified method for calculating the Debye temperature from elastic constants, J. Phys. Chem. Solids 24 (7) (1963) 909-917.

[28] N. W. Ashcroft, N. D. Mermin, Solid State Physics, Harcourt Brace Jovanovich College Publishers, 1976.

[29] F. D. Vila, J. J. Rehr, H. H. Rossner, H. J. Krappe, Theoretical x-ray absorption Debye-Waller factors, Phys. Rev. B 76 (2007) 014301.

[30] G. F. Jenks, The data model concept in statistical mapping, International yearbook of cartography 7 (1967) 186-190.

[31] jenskpy Python module, https://pypi.org/project/jenkspy/

[32] E. Sevillano, H. Meuth, J. Rehr, Extended x-ray absorption fine structure Debye-Waller factors. I. Monatomic crystals, Physical Review B 20 (12) (1979) 4908.

[33] Periodic table of optical constants for the elemental solids, http://feff.phys.washington.edu/optical-constants

[34] Corvus [opcons] and FEFFF installation instructions, http://feff.phys.washington.edu/optical-constants/ installation.html
[35] Corvus [opcons] GitHub repository, https://github.com/ times-software/Corvus/tree/convert-to-3

[36] FEFF10 GitHub repository, https://github.com/ times-software/feff10/tree/fullspectrum

[37] Numpy Python module, https://numpy.org/

[38] Scipy Python module, https://www.scipy.org/

[39] A. Jain, S. P. Ong, G. Hautier, W. Chen, W. D. Richards, S. Dacek, S. Cholia, D. Gunter, D. Skinner, G. Ceder, K. A. Persson, Commentary: The materials project: A materials genome approach to accelerating materials innovation, APL Mater. 1 (2013) 011002. 